\title{
Upfront high-dose chemotherapy combined with autologous stem cell transplantation: Potential survival benefit for patients with high-risk diffuse large B-cell lymphoma
}

\author{
AYA NAKAYA, SHINYA FUJITA, ATSUSHI SATAKE, TAKAHISA NAKANISHI, \\ YOSHIKO AZUMA, YUKIE TSUBOKURA, MASAAKI HOTTA, HIDEAKI YOSHIMURA, \\ KAZUYOSHI ISHII, TOMOKI ITO and SHOSAKU NOMURA
}

First Department of Internal Medicine, Kansai Medical University, Hirakata, Osaka 573-1010, Japan

Received February 9, 2016; Accepted March 9, 2017

DOI: $10.3892 / \mathrm{ol} .2017 .6589$

\begin{abstract}
In patients with diffuse large B-cell lymphoma (DLBCL) classified as high-intermediate risk or high risk using the International Prognosis Index, the efficacy of high-dose chemotherapy combined with upfront autologous stem cell transplantation (HDT/ASCT) remains controversial in the rituximab era. In the present study, 27 patients who had been treated with HDT/ASCT in an upfront setting were retrospectively analyzed, and compared with 77 patients with similar characteristics who had received conventional chemotherapy without HDT/ASCT (the non-upfront setting). The 3-year overall survival and progression-free survival rates in the upfront setting were $88.5 \%(\mathrm{P}=0.0134$ vs. non-upfront setting) and $68.4 \%(\mathrm{P}=0.113$ vs. non-upfront setting), respectively; in the non-upfront setting, the 3 -year overall survival and progression-free survival rates were 60.8 and $50.6 \%$, respectively. In conclusion, the results indicate that upfront HDT/ASCT in patients with high-risk DLBCL is feasible and may improve the outcome of these patients. It may be beneficial for patients to undergo HDT/ASCT as an early treatment, prior to the development of therapy resistance.
\end{abstract}

\section{Introduction}

In patients with diffuse large B-cell lymphoma (DLBCL), rituximab-containing chemotherapy regimens can achieve superior long-term progression-free survival (PFS) and overall survival (OS) rates relative to regimens that do not contain rituximab. However, even in the rituximab era, the survival rates of patients classified as high-intermediate risk or high

Correspondence to: Dr Aya Nakaya, First Department of Internal Medicine, Kansai Medical University, 2-5-1 Shinmachi, Hirakata, Osaka 573-1010, Japan

E-mail: nakaya1016@yahoo.co.jp

Key words: upfront, autologous stem cell transplantation, diffuse large B-cell lymphoma, high-dose chemotherapy, rituximab risk according to the International Prognostic Index (IPI) (1) remain unsatisfactory $(2,3)$. Consequently, several randomized control trials (RCTs) (4-8) have prospectively evaluated the role of upfront autologous stem cell transplantation (ASCT) following therapy with rituximab plus cyclophosphamide, doxorubicin, vincristine and prednisone (R-CHOP) for high-risk DLBCL. Upfront ASCT was performed as a consolidation treatment, and part of first-line treatment with induction chemotherapy. However, although certain studies have reported good outcomes following treatment with upfront high-dose chemotherapy (HDT)/ASCT, its usefulness has not been confirmed in RCTs (4-7). Thus, its significance in the upfront setting remains to be elucidated, and the majority of guidelines recommend HDT/ASCT as salvage therapy. However, in actual clinical practice, there is a dilemma regarding the timing of ASCT in high-risk DLBCL patients who have achieved a complete response (CR) or partial response (PR) following induction therapy: It is unclear whether these patients should undergo upfront HDT/ASCT, or wait until they relapse and subsequently undergo salvage HDT/ASCT.

The aims of the present retrospective study were to evaluate the treatment and outcomes of high-risk DLBCL patients who had received HDT/ASCT, and to identify the clinical factors that define the patients who achieve improved outcomes following upfront HDT/ASCT.

\section{Materials and methods}

Patients. DLBCL patients diagnosed between January 2006 and December 2013 at Kansai Medical University Hospital (Hirakata, Japan) were selected from the hospital database. Overall, the clinical data of 278 patients were collected. Among them, 66 patients were excluded as they were aged $\geq 75$ years, and were ineligible for HDT/ASCT. From the beginning, primary central nervous system lymphoma was not included as its regimen differed from the standard R-CHOP regimen. Overall, 212 patients aged $<75$ years were analyzed. Risk category was identified according to the IPI at initial diagnosis. The age-adjusted (aa) IPI was used for patients aged $<60$ years. High-risk patients included those with high-intermediate-risk or high-risk tumors, while low-risk patients include those with 
low-risk and low-intermediate-risk tumors according to the aaIPI/IPI. Patients who underwent HDT/ASCT were those who were aged $<75$ years in the high-risk group, with good performance status and no severe organ dysfunction. Eligible patients were almost all recommended to undergo HDT/ASCT at the beginning of therapy; however, certain patients refused treatment due to family or economic issues and other factors. Ineligible patients were those with an Eastern Cooperative Oncology Group performance status of 3 or 4 , concomitant disease, previous malignancy or major organ dysfunction. Upfront HDT/ASCT was performed for patients who achieved a first CR or PR following R-CHOP. High-risk patients who did not undergo HDT/ASCT received 4-8 cycles of R-CHOP. Low-risk patients received R-CHOP or R-CHOP in combination with radiotherapy, in accordance with the recommended guidelines. Patients with stable disease or progressive disease following R-CHOP, or who exhibited relapse, underwent salvage therapy, including salvage HDT/ASCT (Fig. 1).

The present study was approved by the Institutional Review Board of Kansai Medical University (Hirakata, Japan). According to the Ethical Guidelines for Medical and Health Research Involving Human Subjects by the Ministry of Health, Labor and Welfare (9), the present study was a retrospective study and did not require informed consent from individual patients. However, the data of the present study was made available through the website (10) and opportunities were established for the patients to refuse.

Staging and response criteria. Clinical staging was performed using positron emission tomography or computed tomography scanning of the neck, thorax, abdomen and pelvis; bone marrow biopsy; cerebrospinal fluid examination; and other tools, such as magnetic resonance imaging, if indicated. The criteria for evaluating the efficacy of treatment have been described by Cheson et al (11).

Induction therapy. The induction therapy regimen consisted of R-CHOP (375 mg rituximab/m² intravenously (i.v), plus $750 \mathrm{mg}$ cyclophosphamide/ $\mathrm{m}^{2}$ i.v, $50 \mathrm{mg}$ doxorubicin $/ \mathrm{m}^{2}$ i.v and $2 \mathrm{mg}$ vincristine $/ \mathrm{m}^{2}$ i.v on day 1 , and $100 \mathrm{mg}$ prednisone $/ \mathrm{m}^{2}$ orally on days 1 through 5) or R-CHOP-like regimens (for patients with poor cardiac function, doxorubicin may be removed or replaced with pinorubicin). In the upfront setting, the treatment response was evaluated after 4-5 cycles of R-CHOP, and peripheral blood stem cell collection was performed using an additional cycle of R-CHOP plus etoposide as a harvest regimen (an established regimen for mobilizing hematopoietic stem cells into the peripheral blood). In the non-upfront setting, patients underwent 4-8 cycles of R-CHOP. Patients with refractory or relapsed disease were treated with salvage regimens consisting of rituximab, etoposide, ifosfamide and dexamethasone (R-DeVIC), or rituximab, etoposide, methylprednisolone, cytarabine and cisplatin (R-ESHAP).

HDT. The day of transplantation was set at day 0 , and the conditioning regimen was initiated counting back prior to transplantation day. Patients undergoing HDT/ASCT received the following conditioning regimen: Ranimustine (also known as MCNU), $300 \mathrm{mg} / \mathrm{m}^{2}$ on day -6 (6 days before ASCT); etoposide, $200 \mathrm{mg} / \mathrm{m}^{2}$ on days -5 to -2 ; cytarabine, $200 \mathrm{mg} / \mathrm{m}^{2}$ on days -5 to -2 ; and melphalan, $140 \mathrm{mg} / \mathrm{m}^{2}$ on day -1 . This regimen (MEAM) is a modified BEAM regimen in which carmustine (BCNU) is replaced with MCNU due to a lack of accessibility of the former in Japan. MEAM is one of the most frequently used conditioning regimens for the treatment of lymphoid malignancies in Japan $(12,13)$.

Supportive care. Bacterial, fungal, herpes simplex virus and pneumocystis pneumonitis prophylaxes were administered to all of the patients in accordance with the guidelines $(14,15)$. In patients undergoing HDT/ASCT, granulocyte colony-stimulating factor was administered intravenously from day +1 until neutrophil recovery.

Statistical analysis. OS was measured from the time of ASCT or the final chemotherapy until the time of mortality from any cause or the final date of observation. PFS was measured from the time of ASCT or the final chemotherapy until the time of disease relapse or progression, mortality from any cause, or the final date of observation. Survival estimates were calculated using the Kaplan-Meier method, and the log-rank test was used for univariate comparisons. To identify the clinical factors that defined the high-risk DLBCL patients who had an improved outcome from upfront HDT/ASCT, univariate analysis and the log-rank test were used. All P-values were two-sided, with $\mathrm{P}<0.05$ considered to indicate a statistically significant difference.

All statistical analyses were performed using EZR (Saitama Medical Center, Jichi Medical University, Saitama, Japan), a graphical user interface for R (version 2.13.0; The R Foundation for Statistical Computing, Australia); EZR is a modified version of $\mathrm{R}$ commander (version 1.6-3) designed to include the statistical functions frequently used in biostatistics (16).

\section{Results}

Patient characteristics. A total of 212 patients were divided into low-risk $(n=108)$ or high-risk groups $(n=104)$ according to the aaIPI/IPI. Among the high-risk patients, 27 underwent upfront HDT/ASCT and 77 received conventional chemotherapy. Of the 212 patients, 79 experienced relapse; there were 33 low-risk patients, 10 patients treated in an upfront setting involving HDT/ASCT and 36 patients treated in a non-upfront setting. Among the 79 relapsed patients, 60 patients (including 10 who had relapsed subsequent to upfront HDT/ASCT) received salvage chemotherapy, and 19 received HDT/ASCT as salvage therapy. These patients treated with HDT/ASCT received 3-4 cycles of the salvage regimens following relapse; 16 of these patients achieved a second CR, 1 patient achieved a PR, and 2 patients suffered disease progression (Fig. 1).

Table I shows the baseline characteristics of the high-risk patients: 27 of these patients received upfront HDT/ASCT and 77 received conventional R-CHOP without HDT/ASCT. The median age of the high-risk patients was 62 years in the upfront setting and 67 years in the non-upfront setting. In the upfront setting, all patients were categorized as high-intermediate or high risk according to the IPI, and as clinical stage III or IV. Elevated lactate dehydrogenase (LDH) levels were observed more frequently in the upfront setting, whereas the incidence of extranodal disease was similar to that in the non-upfront setting. 
Table I. Characteristics of the high-risk patients $(n=104)$ who received upfront HDT/ASCT and those who did not (non-upfront).

\begin{tabular}{lcc}
\hline Characteristic & Upfront & Non-upfront \\
\hline Total patients (n) & 27 & 77 \\
Age (years) & & \\
$\quad$ Median & 62 & 67 \\
Range & $36-72$ & $20-75$ \\
Sex (\%) & & \\
Male & 56 & 65 \\
Female & 44 & 35 \\
IPI/aaIPI $(\%)$ & & \\
High-intermediate & 37 & 44 \\
High & 63 & 56 \\
Stage (\%) & & \\
I & 0 & 5 \\
II & 0 & 69 \\
III & 26 & 69 \\
IV & 74 & 58 \\
Bulky mass (\%) & 7 & 68 \\
Extranodal involvement & & \\
at $>1$ site $(\%)$ & 56 & \\
Elevated LDH level $(\%)$ & 81 & \\
HDT/ASCT as salvage $(\%)$ & 0 & \\
\hline
\end{tabular}

IPI, International Prognostic Index; aaIPI, age-adjusted IPI; LDH, lactate dehydrogenase; HDT/ASCT, high-dose chemotherapy with autologous stem cell transplantation.

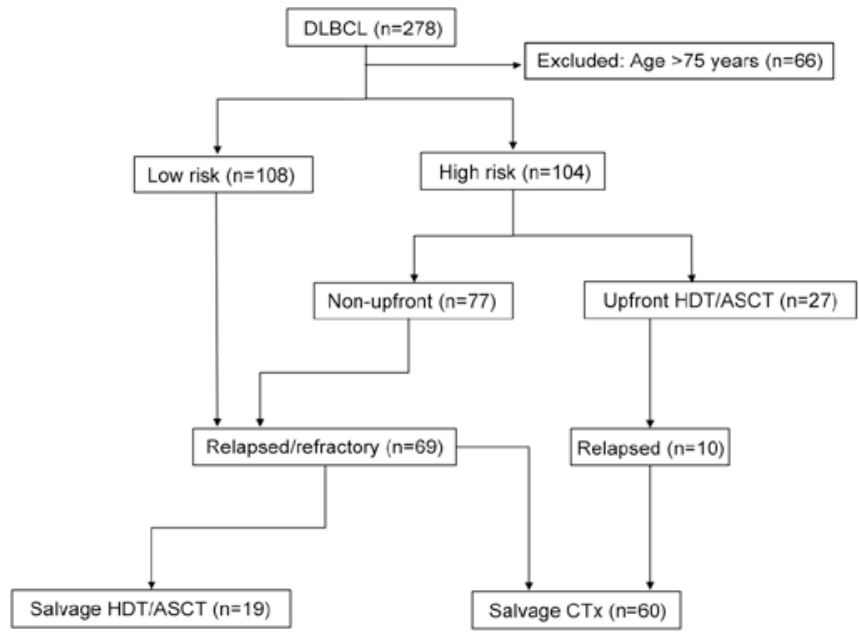

Figure 1. Flow diagram of 278 patients with DLBCL included in the present study. DLBCL, diffuse large B-cell lymphoma; HDT/ASCT, high-dose chemotherapy with autologous stem cell transplantation; CTx, chemotherapy.

Table II shows the baseline characteristics of the 79 relapsed patients. A total of 19 patients received salvage HDT/ASCT and 60 received salvage chemotherapy. Patient median age was 58 years in the salvage HDT/ASCT setting, which was lower than that in the salvage chemotherapy group, which is the group
Table II. Characteristics of the relapsed patients $(n=79)$.

\begin{tabular}{|c|c|c|}
\hline Characteristic & $\begin{array}{c}\text { Salvage } \\
\text { HDT/ASCT }\end{array}$ & $\begin{array}{c}\text { Salvage } \\
\text { chemotherapy }\end{array}$ \\
\hline Total patients (n) & 19 & 60 \\
\hline \multicolumn{3}{|l|}{ Age (years) } \\
\hline Median & 58 & 67 \\
\hline Range & $36-65$ & $37-75$ \\
\hline \multicolumn{3}{|l|}{$\operatorname{Sex}(\%)$} \\
\hline Male & 74 & 55 \\
\hline Female & 26 & 45 \\
\hline \multicolumn{3}{|l|}{ IPI/aaIPI (\%) } \\
\hline Low & 32 & 22 \\
\hline Low-intermediate & 37 & 17 \\
\hline High-intermediate & 0 & 33 \\
\hline High & 31 & 28 \\
\hline \multicolumn{3}{|l|}{ Stage $(\%)$} \\
\hline I & 11 & 13 \\
\hline II & 32 & 7 \\
\hline III & 37 & 20 \\
\hline IV & 20 & 60 \\
\hline Bulky mass (\%) & 0 & 3 \\
\hline $\begin{array}{l}\text { Extranodal involvement } \\
\text { at }>1 \text { site }(\%)\end{array}$ & 21 & 63 \\
\hline Elevated LDH level (\%) & 21 & 63 \\
\hline
\end{tabular}

HDT/ASCT, high-dose chemotherapy with autologous stem cell transplantation; IPI, International Prognostic Index; aaIPI, age-adjusted IPI; LDH, lactate dehydrogenase.

of patients whom relapsed and resisted the initial treatment and received chemotherapy without HDT/ASCT. Factors reflecting tumor burden, namely bulky mass, extranodal DLBCL and elevated LDH, were observed more often in the salvage chemotherapy patient group than in the salvage ASCT patient group.

Treatment efficacy and prognosis. In the high-risk group, the 3 -year OS rates in the upfront and non-upfront settings were $88.5 \%$ [95\% confidence interval (CI), 68.4-96.1\%] and $60.8 \%$ (95\% CI, 48.3-71.2\%), respectively ( $\mathrm{P}=0.0134$; Fig. 2A). The 3 -year PFS rates in the upfront and non-upfront settings were 68.4\% (95\% CI, 46.4-82.9\%) and 50.6\% (95\% CI, 38.3-61.6\%), respectively ( $\mathrm{P}=0.113$; Fig. $2 \mathrm{~B})$. In the relapsed group, the 3 -year OS rates in the salvage HDT/ASCT and the salvage chemotherapy groups were $57.9 \%(95 \% \mathrm{CI}, 33.2-76.3 \%)$ and $33.5 \%$ (95\% CI, 21.7-45.7\%), respectively ( $\mathrm{P}=0.00193$; Fig. 3A). The 3 -year PFS rates in the salvage HDT/ASCT and the salvage chemotherapy groups were $36.8 \%$ (95\% CI, 16.5-57.5\%) and $19.4 \%$ (95\% CI, 10.4-30.3\%), respectively ( $\mathrm{P}=0.0532$; Fig. 3B).

High-dose regimen-related toxicity and treatment-related mortality. Common hematological regimen-related toxicities (RRTs), comprising neutropenia, anemia and thrombocytopenia at grades 3 and 4, were observed in all patients. The most common non-hematological RRTs were anorexia and nausea 

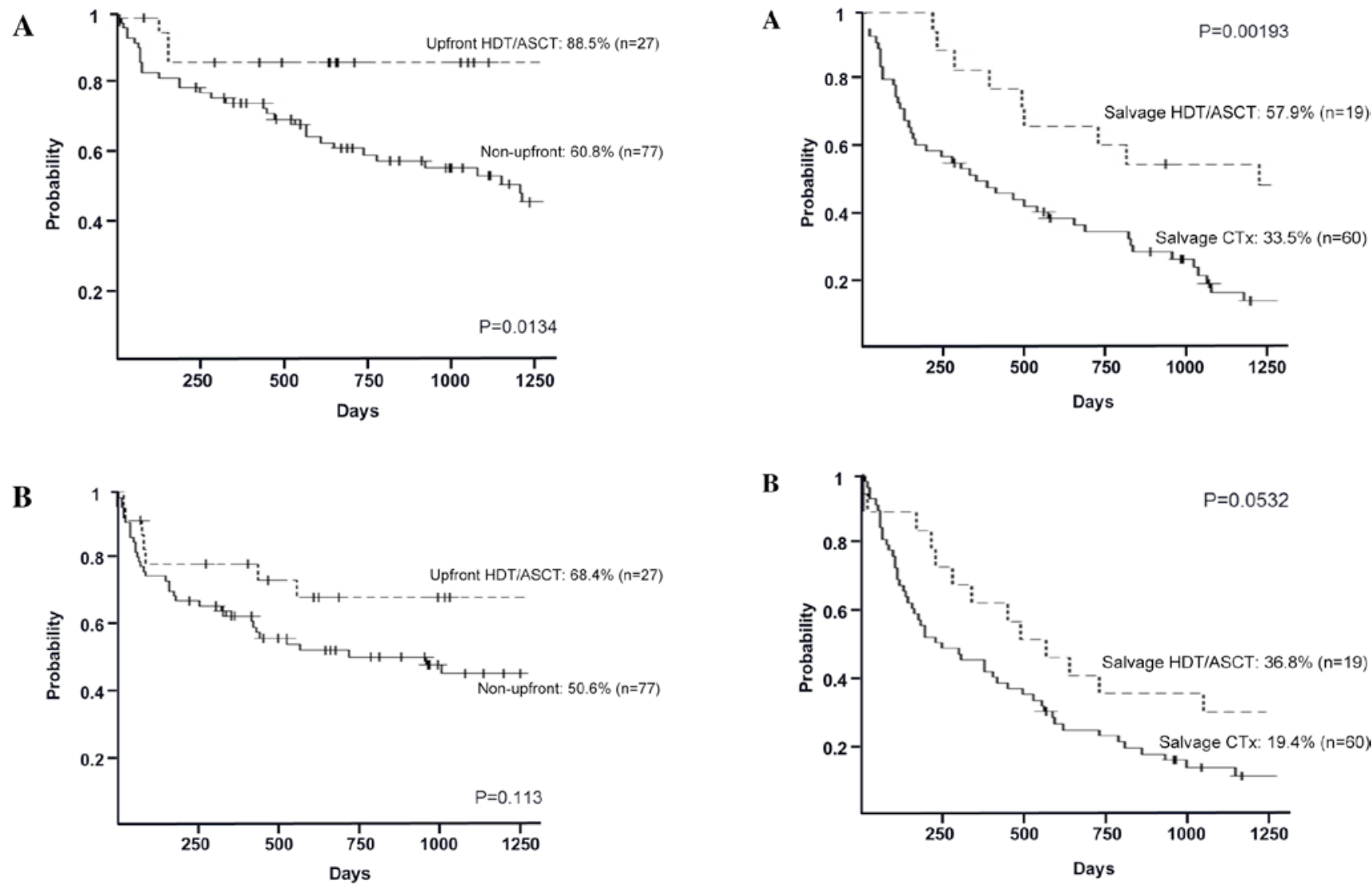

Figure 2. Kaplan-Meier survival curves for the comparison of high-risk patients with diffuse large B-cell lymphoma who received upfront HDT/ASCT (n=27) and those who did not (non-upfront; n=77). (A) 3-year overall survival rates were 88.5 and $60.8 \%$ in the upfront and non-upfront settings, respectively $(\mathrm{P}=0.0134)$. (B) 3-year progression-free survival rates were 68.4 and $50.6 \%$ in the upfront and non-upfront settings, respectively $(\mathrm{P}=0.113)$. HDT/ASCT, high-dose chemotherapy with autologous stem cell transplantation.

at grades 3 and 4, which were noted in all patients. Diarrhea and stomatitis were also commonly observed (80-90\% of patients). Mucosal damage was relatively severe in the salvage HDT/ASCT group. Grade 2 rash was encountered in both upfront and salvage HDT/ASCT groups. Febrile neutropenia (FN) was observed in $96 \%$ of patients in the upfront setting and $55 \%$ in the salvage setting. In the salvage setting, 1 patient succumbed to FN that occurred prior to engraftment. Another notable complication was interstitial pneumonia: In the salvage group, 1 patient died as a result of rapid onset of interstitial pneumonia. The overall rate of treatment-related mortality (TRM) was $4.3 \%$.

Univariate analysis. To identify the clinical factors that define high-risk DLBCL patients who can achieve improved outcomes from upfront HDT/ASCT, a univariate analysis for 3 -year OS rate was performed. There were no statistically significant prognostic factors identified in the upfront setting; a hemoglobin level $<11 \mathrm{~g} / \mathrm{dl}$ was associated with a lower OS rate, without statistical significance in this setting $(\mathrm{P}=0.074)$. A high LDH level revealed a significant association with survival rate in the non-upfront setting $(\mathrm{P}=0.024)$. Although there were no statistically differences observed, patients aged $>65$ years

Figure 3. Kaplan-Meier survival curves for the comparison of patients with relapsed/refractory diffuse large B-cell lymphoma who received salvage HDT/ASCT $(n=19)$ or salvage CTx $(n=60)$. (A) 3-year overall survival rates were 57.9 and $33.5 \%$ in the salvage HDT/ASCT and salvage CTx groups, respectively $(\mathrm{P}=0.00193)$. (B) 3-year progression-free survival rates were 36.8 and $19.4 \%$ in the salvage HDT/ASCT and salvage CTx groups, respectively $(\mathrm{P}=0.0532)$. HDT/ASCT, high-dose chemotherapy with autologous stem cell transplantation; CTx, chemotherapy.

and those with extranodal disease exhibited poorer OS rates in the non-upfront setting (Table III).

\section{Discussion}

The findings of the present retrospective study demonstrated the efficacy of upfront HDT/ASCT in patients with high-risk DLBCL. While certain studies have reported good outcomes regarding upfront HDT/ASCT $(4,6,8)$, its usefulness has not been confirmed in RCTs. In terms of the 2-year OS rate, Vitolo et al (4) found no significant differences between the study arms in which patients were treated with R-CHOP alone or R-CHOP followed by HDT/ASCT; however, the 2-year PFS rate was significantly higher in the HDT/ASCT arm (4). Stiff et al (8) reported that in the subset of high-risk patients alone, induction chemotherapy followed by early HDT/ASCT significantly improved 2-year PFS and OS rates relative to chemotherapy alone. Based on this report, as of 2015, the National Comprehensive Cancer Network Guidelines include HDT/ASCT as one of the optional therapies following R-CHOP for high-risk patients who achieved a CR following induction therapy (17). However, at present, most guidelines recommend HDT/ASCT as salvage therapy $(15,17)$. 
Table III. Univariate analysis for 3-year OS rate in high-risk patients.

\begin{tabular}{|c|c|c|c|c|}
\hline \multirow[b]{2}{*}{ Characteristic } & \multicolumn{2}{|c|}{ Upfront } & \multicolumn{2}{|c|}{ Non-upfront } \\
\hline & 3 -year OS rate $(\%)$ & $\mathrm{P}$-value & 3-year OS rate $(\%)$ & P-value \\
\hline Age (years) & & 0.234 & & 0.393 \\
\hline$\leq 65$ & 83.3 & & 62.1 & \\
\hline$>65$ & 100.0 & & 60.2 & \\
\hline Sex & & 0.425 & & 0.735 \\
\hline Female & 90.9 & & 65.5 & \\
\hline Male & 85.7 & & 58.2 & \\
\hline Alb (g/dl) & & 0.386 & & 0.527 \\
\hline$>3.5$ & 88.9 & & 61.5 & \\
\hline$\leq 3.5$ & 87.5 & & 60.6 & \\
\hline LDH & & 0.133 & & 0.024 \\
\hline No & 80.0 & & 86.2 & \\
\hline Yes & 90.5 & & 50.9 & \\
\hline Hemoglobin (g/dl) & & 0.074 & & 0.486 \\
\hline$>11$ & 94.7 & & 59.0 & \\
\hline$\leq 11$ & 71.4 & & 64.9 & \\
\hline Extranodal disease & & 0.591 & & 0.668 \\
\hline No & 81.8 & & 70.6 & \\
\hline Yes & 93.3 & & 57.2 & \\
\hline
\end{tabular}

OS, overall survival; LDH, lactate dehydrogenase.; Alb, albumin.

In the present study, a significant difference in the 3-year OS rate was identified between the upfront ASCT setting and the non-upfront setting: The 3-year OS rate in the upfront setting was $88.5 \%$ ( $\mathrm{P}=0.0134$ vs. non-upfront setting), which was higher than the 3 -year OS rate reported in previous studies (4-8). However, there was no statistically significant difference in the 3-year PFS rate between the upfront and non-upfront groups, indicating that the high-risk patients may eventually relapse, even after upfront HDT/ASCT. The 3-year OS and PFS rates in the non-upfront setting were 60.8 and $50.6 \%$, respectively, and were significantly lower than those in the upfront setting ( $\mathrm{P}=0.113$ for PFS rate). These results confirmed that upfront HDT/ASCT for high-risk DLBCL is a feasible and promising therapy.

As the majority of guidelines recommend HDT/ASCT as salvage therapy, a comparison between salvage HDT/ASCT and salvage chemotherapy was performed in the present study. The 3-year OS rate of the salvage HDT/ASCT group was $57.9 \%(\mathrm{P}=0.00193)$, which is unsatisfactory in comparison with the OS rate of the upfront HDT/ASCT group. The 3-year PFS rate in this setting was $36.8 \%(\mathrm{P}=0.0532)$, which revealed that relapse was frequent even after salvage HDT/ASCT. These results indicate that DLBCL is frequently resistant to chemotherapy following relapse. The patients in the salvage HDT/ASCT group were younger on average than those in the salvage chemotherapy group; this indicates that patients who are able to undergo salvage HDT/ASCT must be limited due to age or co-morbidity. Thus, the timing of HDT/ASCT administration is critical. If patients are eligible, it would be beneficial for them to undergo HDT/ASCT as an early treatment before they become therapy-resistant or more elderly with more complications.

In the present study, the common RRTs were anorexia, nausea, diarrhea, stomatitis and FN. In total, 2 patients died due to interstitial pneumonia or $\mathrm{FN}$; as these 2 patients were treated in the salvage setting, potential organ damage caused by prior chemotherapies may have caused these lethal adverse effects. However, according to the present results, the TRM rate was low (4.3\%); thus, we suggest that there is no reason to avoid HDT following ASCT if patients are eligible.

HDT/ASCT may be tolerable and effective; however, serious adverse effects can occur. Therefore, it is necessary to identify patient groups that will gain the maximum benefit from upfront HDT/ASCT. In this regard, the most accurate prognostic factors must be determined. Using univariate analysis, the present study did not reveal any significant prognostic factors in the upfront setting. Notably, patients in the upfront setting with advanced age (>65 years), elevated LDH levels or extranodal disease had a better prognosis than younger patients, those with normal LDH, or those without extranodal disease, respectively; this suggests that HDT/ASCT may overcome the unfavorable outcomes caused by these prognostic factors.

The present study had a number of limitations. First, a small patient cohort was evaluated and the follow-up period was short. To determine the true efficacy of HDT/ASCT, a longer follow-up period will be required. Furthermore, biological features, such as genetic abnormalities or CD5 
expression, were not considered. These factors are expected to be important in the prediction of survival. In the present study, the 3-year OS and PFS rates were superior to those previously reported for the following reasons. First, as our institute is a university hospital, patients had already been selected before they attended our out-patient department; consequently, there would have been a selection bias. Second, it is suspected that the use of the IPI will fail in the classification of appropriate patients for upfront HDT/ASCT; it has been reported that the IPI cannot be used to predict the outcome of a patient group with poor prognosis where the 5-year OS rate is $<60 \%$ (3). Thus, the population must have included patients with a good prognosis who did not require upfront treatment with HDT/ASCT. Therefore, it is necessary to establish prognostic factors additional to the IPI that can be used to identify the patients who will benefit from upfront HDT/ASCT. In the current study, no significant prognostic factors associated with upfront HDT/ASCT could be determined. A sufficiently large study population will be required to provide the statistical power to adequately assess these factors, and prospective studies will be required to confirm the efficacy of upfront HDT/ASCT.

In conclusion, the use of upfront HDT/ASCT in patients with high-risk DLBCL is feasible and may improve their outcome. HDT/ASCT should be administered as an early treatment before patients become therapy-resistant.

\section{References}

1. A predictive model for aggressive non-Hodgkin's lymphoma The International Non-Hodgkin's Lymphoma Prognostic Factors Project. N Engl J Med 329: 987-994, 1993.

2. Ziepert M, Hasenclever D, Kuhnt E, Glass B, Schmitz N, Pfreundschuh M and Loeffler M: Standard international prognostic index remains a valid predictor of outcome for patients with aggressive CD20+ B-cell lymphoma in the rituximab era. J Clin Oncol 28: 2373-2380, 2010.

3. Sehn LH, Berry B, Chhanabhai M, Fitzgerald C, Gill K, Hoskins P, Klasa R, Savage KJ, Shenkier T, Sutherland J, et al: The revised International Prognostic Index (R-IPI) is a better predictor of outcome than the standard IPI for patients with diffuse large B-cell lymphoma treated with R-CHOP. Blood 109: 1857-1861, 2007.

4. Vitolo U, Chiappella A, Brusamolino E, et al: A randomized multicenter phase III study for first line treatment of young patients with high risk (AAIPI 2-3) diffuse large B-cell lymphoma (DLBCL): Rituximab (R) plus dose-dense chemotherapy CHOP14/MEGA CHOP14 with or without intensified high-dose chemotherapy (HDT) and autologous stem cell transplantation (ASCT). Results of DLCL04 trial of Italian lymphoma foundation (FIL). Ann Oncol 22: 106, 2011
5. Schmitz N, Nickelsen M, Ziepert M, Haenel M, Borchmann P, Schmidt C, Viardot A, Bentez N, Peter N, Ehninger G, et al: Conventional chemoimmunotherapy (R-CHOEP)-14 or high-dose therapy (R-Mega-CHOEP) for young, high-risk patients with aggressive B-cell lymphoma: Final results of the randomized Mega-CHOEP trial of the German High-Grade Non-Hodgkin Lymphoma Study Group (DSHNHL). J Clin Oncol 29: 8002, 2011.

6. Stiff PJ, Unger JM, Cook J, Constine S, Couban S, Shea TC, Winter JN, Miller TP, Tubbs RR, Marcellus DC, et al: Randomized phase III U.S./Canadian intergroup trial (Swog S9704) comparing CHOP (+/-) R for eight cycles to CHOP (+/-) R for six cycles followed by autotransplantation for patients with high-intermediate (H-int) or high IPI grade diffuse aggressive non-Hodgkin lymphoma (NHL). J Clin Oncol 29: 8001, 2011

7. Le Gouill S, Milpied NJ, Lamy T, Delwail V, Gressin R, Guyotat D, Damaj GL, Foussard C, Cartron G, Maisonneuve $\mathrm{H}$, et al: First-line rituximab (R) high-dose therapy (R-HDT) versus R-CHOP14 for young adults with diffuse large B-Cell lymphoma: Preliminary results of the GOELAMS 075 prospective multicenter randomized trial. J Clin Oncol 29: 8003, 2011.

8. Stiff PJ, Unger JM, Cook JR, Constine LS, Couban S, Stewart DA, Shea TC, Porcu P, Winter JN, Kahl BS, et al: Autologous transplantation as consolidation for aggressive non-Hodgkin's lymphoma. N Engl J Med 369: 1681-1690, 2013.

9. The Ministry of Health, Labor and Welfare. Ethical Guidelines for Medical and Health Research Involving Human Subjects. http://www.mhlw.go.jp/file/06-Seisakujouhou-10600000-Daijinkanboukouseikagakuka/0000080278.pdf

10. http://www.kmu.ac.jp/hirakata/visit/treatment/medical_ section/ketuekishuyounaika.html

11. Cheson BD, Horning SJ, Coiffier B, Shipp MA, Fisher RI, Connors JM, Lister TA, Vose J, Grillo-López A, Hagenbeek A, et al: Report of an international workshop to standardize response criteria for non-Hodgkin's lymphomas. NCI Sponsored International Working Group. J Clin Oncol 17: 1244, 1999.

12. Kameoka Y, Takahashi N, Ishizawa K, Kato Y, Ito J, Sasaki O, Murai K, Noji H, Hirokawa M, Tajima K, et al: Safety and feasibility of high-dose ranimustine (MCNU), carboplatin, etoposide and cyclophosphamide (MCVC) therapy followed by autologous stem cell transplantation for malignant lymphoma. Int J Hematol 96: 624-630, 2012.

13. Kawabata K, Hagiwara S, Takenouchi A, Tanimura A, Tanuma J, Tachikawa N, Miwa A and Oka S: Autologous stem cell transplantation using MEAM regimen for relapsed AIDS-related lymphoma patients who received highly active anti-retroviral therapy: A report of three cases. Intern Med 48: 111-114, 2009.

14. NCCN Clinical Practice Guidelines in Oncology: Prevention and Treatment of Cancer-Related Infections, Version 1. 2017. https://www.nccn.org/professionals/physician_gls/f_guidelines. asp

15. Japanese Society of Hematology, clinical practice guideline. http://www.jshem.or.jp/gui-hemali/table.html

16. Kanda Y: Investigation of the freely available easy-to-use software 'EZR' for medical statistics. Bone Marrow Transplant 48: 452-458, 2013.

17. NCCN Clinical Practice Guidelines in Oncology: Non-Hodgkin's lymphomas, Version 2. 2016. https://www.nccn.org/professionals/physician_gls/f_guidelines.asp 